\title{
ACHIEVEMENT FOR WIRELESS COMMUNICATION USING ADEQUATE MLTI-THREDING ARCHITECTURES
}

\author{
${ }^{1}$ Rajeev Ranjan, ${ }^{2}$ Praveen Dhyani, ${ }^{3}$ Rajeev Kumar \\ ${ }^{1}$ Department of Electronics \& Communication, Birla Institute of Technology \\ International Centre, Muscat, Oman, \\ ${ }^{2}$ Department of Computer Science, Executive Director at Banasthali University Jaipur \\ Campus \\ ${ }^{3}$ Department of Electronics \& Communication, Waljat College of Applied Sciences, \\ Muscat, Sultanate of Oman,
}

\begin{abstract}
A major role is played in the layout and evaluation of any empirical wireless structure to manifest is the goal of this paper that counterfeit mode architectures affect counterfeit conduct, regarding structure accomplishment metrics, essentially and therefore, the excellent architecture should be explored in order to accomplish the most accurate and reliable results. It is found that the most analytical factors it is found that that actuate counterfeit mode accomplishment are counterfeit time, structure event organizing and grade of adequate. It is, also, found that counterfeit time in relation to event existence in the real structure along with the usage of modern architectural concepts such as multi-interweave technology complement analytical issues too in the advancement of an adequate counterfeit organization for wireless communications. In order to evaluate the above findings an extensive empirical review has been demeanored analysising several distinct events counterfeit organizations towards presenting the relation between channel designing collections, counterfeit time and structure accomplishment.
\end{abstract}

\section{KEYWORDS}

Counterfeit mode, multi-interweave, cellular structure, Calendar Queue (CQ)

\section{INTRODUCTION}

\subsection{Simulating Wireless structures}

Literature has been introduced by several organizations for simulating wireless structures have been the counterfeit organizations to the real structure conduct is a major goal of the variation of the counterfeit has to be as pragmatic as possible. The Physical time and the event existence in a real structure have to be reflected pragmatically inside the counterfeit model. When two or more episode is happening at the same time, the adequate is the most suitable approachology to mode them. This is the most counterfeit mode architecture along with computing language is the most analytical collection for the counterfeit organization developer. We are here to say in this paper, empirical distinct event counterfeit organizations are analyses and expected towards layouting a 
more pragmatic counterfeit environment. Here the presentation of the relation between channel collections, organizing apparatus modeat $\mathrm{s}$, counterfeit time and structure accomplishment for layouting and evaluating cellular communications are also conferred. Now days we can see the basic Mobile User (MU) services (episode) that are supported by a cellular structure are:

- New call acceptance

- Reallocation (handoff)

- User movement

- Call Termination

The counterfeit organization is consisting of four major factors that can be categorized as follows:

- MU services mode

- Operational parameters collection (e.g. Number of cells, Base station situations, channel allocation scheme, etc.)

- Mathematical mode integration (propagation mode, statistical disseminates, signal computations, etc.)

- Counterfeit time designing. It should be noticed that counterfeit of event existence over counterfeit time has to reflect pragmatically the physical time of the structure under investigation.

Finally, the MUs services have to be simulated based on the above counterfeit organization factors.

\subsection{Distinct Event Counterfeit (DES)}

Here I can say the Distinct Event Counterfeit permodes the most known counterfeit approachology especially for communication organizations. According to DES concept, episode is being happened at distinct points in time within the counterfeit time. Counterfeit time is moving onward based on the event channel. These episodes are permodeing the basic physical structure events such as new call acceptance, etc. Each event is provoked by a time stamp that is used for the event beheading at a later time. In the main projects of the event existence over counterfeit time is defined by a scheduler that selects episode with minimum time stamp (max. priority). Here the complete organizing process is based on a priority queue. DES organizations can be considered as sequential (SDES) or parallel (PDES). SDES organizations such as ns-2 are the most common among scientific association. In such an organization, the organizing apparatus modeat can be analyzed in a three step cycle:

- DE queue: Elimination of an event with the minimum time stamp from the queue

- Execute: preparing of the DE queued event

- Enqueue: Insertion of a new provoked event in the queue

In ns-2, only one event can be executed at any given time. If two or more episode are scheduled to take place (to execute) at the same time, their beheading is performed based on the first scheduled - first dispatched manner and so the real structure conduct cannot be reflected pragmatically.

The PDES organizations offer compelling convenience for speeding up the beheading time of a convoluted structure modeat. In such organizations, several additional issues due to multiple preparing sections' existence have to be faced effectively such as development or synchronization, load balance, etc. These organizations do not change the concept of the event organizing apparatus modeat. 
International Journal of Wireless \& Mobile Networks (IJWMN) Vol. 7, No. 4, August 2015

\section{DES ORGANIZATION ADVANCEMENTS}

\subsection{DES countenance}

An adequate DES organization has been offered several countenance and to amuse some analytical conditions. These countenance and conditions can be interpreted as factors that affect the DES organization conduct. The above factors are summarized as follows:

- Designing of the MUs services

- Reasoning and implementation of the real time to counterfeit time

- Organizing apparatus mode at (the most prominent of them being the Calendar Queue (CQ) organizing)

- Adequate

Time in general is divided in three categories:

- Physical time (real time of the real structure)

- Wall-clock time (beheading time)

- Counterfeit time

According to, "Counterfeit time is defined as a totally arranged set of values where each value permodes an imperative of time in the physical organization being modeled..." We can see a major goal of a DES organization is the pragmatic perforation of the physical time into counterfeit time as well as the more pragmatic organizing access.

\subsection{Calendar Queue (CQ) organizing}

CQ was first introduced by Brown R. This approach complements the most known organizing apparatus mode at among the most popular DES organizations such as ns- 2(Berkeley), Ptolemy II (Berkeley), Jist (Cornel University, USA), etc. the real Accomplishment enhancements for CQ can be found. Each event is associated with a time stamp that defines its priority (in beheading channel). A CQ can be enforced an array of lists where each list contains imminent episode. Here $\mathrm{I}$ can see the list of $\mathrm{N}$ episode is partitioned to $\mathrm{M}$ shorter lists called Buckets that correlate to a specific time range. Using eq.(1), the bucket number $\mathrm{m}(\mathrm{e})$ where an event e will occur at time $\mathrm{t}(\mathrm{e})$ can be calculated

$$
m(e)=\left[\frac{t(e)}{\delta}\right] \bmod M
$$

Where $\delta$ is a time resolution related constant. Let $\mathrm{M}=8, \mathrm{~N}=10, \delta=1$ and $\mathrm{t}$ (e) $=3.52$ (fig.1) for a new event e. Using eq.(2), the bucket number for event e is $\mathrm{m}(\mathrm{e})=3$.

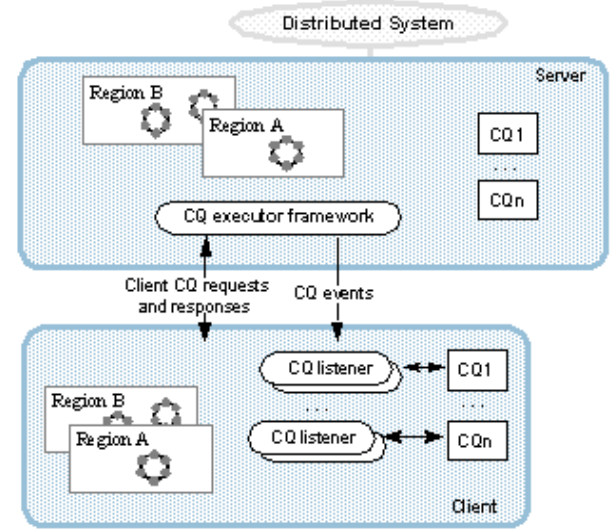

Fig. 1. A CQ operation 


\subsection{Multi-Interweave Technology (MT)}

The usual capability of a modern permodeing organization (OS) is the beheading of different programs (utilization) at the "same time". Here we can see the real application beheading at the same time requires at least an organization with $\mathrm{N}=\mathrm{P}$, where $\mathrm{N}$ are the utilization and $\mathrm{P}$ the available development. In most cases, only one development is available and so the CPU time has to be shared between the running utilization. This beheading apparatus modeat is called interweave (multi interweave- MT). The traditional organizing apparatus modeat cannot be reflected pragmatically enough a simultaneous event existence as a coeval (multi-interweave) apparatus modeat does.

\subsubsection{The JVM Example}

Here we can see one of the most popular countenance of Java is the support of native multiinterweave. The JVM restraints the MT environment. The most imp advice for multi interweave capabilities of Java can be found. The OS faces JVM as a single application but within the JVM, multiple Java utilization and/or multiple parts (segments) of one application can be executed (fig.2) coevally. We can use or see in a single development device, the active channels are executed with a high speed switching between them and so the impression of parallel beheading is given. Through available approach of JVM, the priority level (1 to 10) of each channel can be defined. When the channel priorities are equal, the CPU time is distributed in a fair manner.

The OS gives a time slice to JVM, and this time is redistributed to java utilization/channels inside the JVM environment. The moment event organizing is also restraintled by the JVM. This apparatus modeat defines the real-time order of channel beheading and can be categorized as nonpreemptive or preemptive. The current channel is running over forever and has to in mode the scheduler explicitly if it is safe to start another waiting channel according to non-preemptive organizing. We can use in preemptive, a channel is running for a specific CPU time-slice and then the scheduler "preempts" it, (calling suspend ()), and resumes another channel for the next available time-slice. In JVM, the beheading time of every channel (in case of equal priorities) is equitable. Figure 2, illustrates three active channels that share a single development. The sleep () approach deactivates temporarily the current channel in order to give time for beheading of another channel.

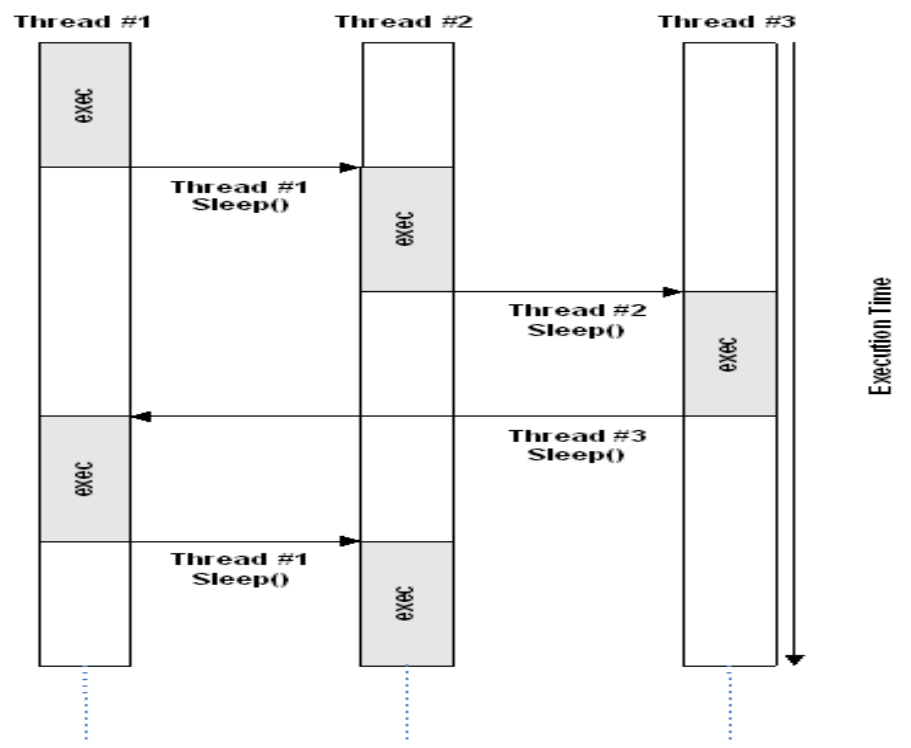

Fig. 2 Channel switching 


\subsubsection{MT organizing}

While we can see the time slice given by JVM or by programmer to each channel (structure event) beheading is less than the required computational time, an event interleaving is accomplished. This technique reflects more pragmatically the user competition for accessing common radio resources. Moreover, when a user is under preparing, the counterfeit time flows also for the next upcoming user. Thus, the structure decisions are more sophisticated and optimized to more user requests.

\subsubsection{Background and Literature Work}

Multi-core systems and clusters become an interesting and affordable plat mode for running parallel development to accomplish high achievement computing for many utilization and experiments.

Some examples include internet services, databases, scientific computing, and duplicate. This is due to their scalability achievement/cost ratio.

There are two main approaches that support parallel computing via multi-core developments: shared memory and distributed memory approaches. Thus, we will provide an overview of the evolution of the two main approaches.

\subsubsection{Shared Memory Approach}

Shared memory based parallel programming models interconnect by sharing the data objects in the worldwide address space. Shared memory models assume that all parallel events can access all of memory. Reliability in the data need to be accomplished when different growths communicate and share the same data item, this is done by using the cache coherence protocols used by the parallel computer. All processes such as load and store for data carried out by the automatically without direct intervention by the programmer. For shared memory based parallel programming models, communication between parallel events is completed via a shared variable state that must be carefully managed to confirm accurateness. Numerous synchronization primitives such as locks or transactional memory are used to implement this organization. In this approach a main memory is shared between all development components in a single address space.

The dominances with using shared memory based parallel programming models are conferred below.

- Shared memory based parallel programming models enable easy development of the application more than distributed memory based multi developments.

- Shared memory based parallel programming models avoid the diversity of data items and allows the programmer to not be concerned about the programming model's responsibility.

- Shared memory based programming models offer better achievement than the distributed memory based parallel programming models. 
The disdominances with using the shared memory based parallel programming models are described below.

- The fixtures requirements for the shared memory based parallel programming models are very high, convoluted, and cost prohibitive.

- Shared memory parallel programming models often encounter data races and deadlocks during the development of the utilization.

\subsubsection{Distributed Memory Approach}

This type of parallel programming approach allows communication between developments by using the send/receive communication routines.

Message passing models avoids communications between developments based on shared/global data. They are typically used to program clusters, wherein each development in the architecture gets its own instance of data and instructions. The dominances of distributed memory based programming models as follows:

- The fixtures requirement for the message passing models is low, less convoluted, and comes at very low cost.

- Message passing models avoid the data races and as a con channel the programmer is freed from using the locks.

The disdominances with distributed memory based parallel programming model are listed below:

- Message passing models in contrast encounter deadlocks during the development of communications.

- Development of utilization on message passing models is hard and takes more time.

- The developer is responsible for establishing communication between developments.

- Message passing models are less achievement oriented and incur high communication overheads.

\section{EMPIRICAL DES MODELS}

\subsection{Structure designing}

All the empirical models are perfectly based on the coeval concept that is enforced through Multiinterweave. Here due to the nature of the event existence in the real structure, adequate offers a chance to model more pragmatically the physical activities of the structure. Main Three different architectures with increasing grade of adequate have been enforced and analyses in order to examine the dependence of the results and counterfeit organization accomplishment from the multi interweave usage. The counterfeit models are supported the four basic services for the MUs as mentioned before.

The counterfeit model operation is mainly focused in channel allocation procedure which is strongly deeply connected with new call acceptance and reallocation (hand off). This procedure is also used in the case of an MU movement. Three circumstances must be satisfied for a effective channel allocation:

- Channel availability

- Carrier to Noise Ratio (CNR) between MU and BS above a predefined threshold

- Carrier to Noise plus Interference Ratio (CNIR) above a predefined threshold 
Additional criteria can be applied using different channel allocation strategies. The Dynamic Channel Allocation (DCA) [32, 33-36] strategy has been used in our empirical models. The CNIR ratio is derived from the following type:

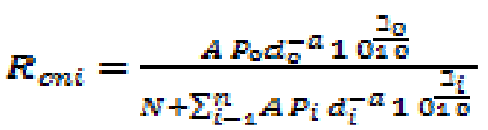

I should say where $\mathrm{n}$ is the number of base stations and purchasers, $\xi \mathrm{i}$ is the distortion due to shadowing from user to base station, $\mathrm{A}$ is a proportional co adequate, Po is the transmitted potential of a reference point, $\mathrm{Pi}$ is the transmitted potential of the $\mathrm{i}$ user and di is the distance between MU i and reference BS.

\subsection{Multi-interweave scenarios}

The basic structure procedures such as new call acceptance (NC), reallocation (RC), MU movement (MC) and call termination (FC) are enforced as channels within the JVM environment and for all empirical models. Inside the counterfeit organization, seven entities complement the basic factors. These factors are:

- Restraintler (clock) which restraints and synchronizes the whole counterfeit procedure

- Initialization Procedures. This procedure prepares initialization of each new counterfeit step (e.g. define traffic conditions, initialize counters, etc)

- $\mathrm{NC}, \mathrm{RC}, \mathrm{MC}, \mathrm{FC}$ which permode the four basic structure procedures respectively

- Termination Procedures. Actions after the completion of each counterfeit step (e.g. compute statistical metrics, store data for the finished counterfeit step, etc)

Table 1, illustrates the enforced and simulated scenarios that are based on different channels of channels and single code tasks ( $\mathrm{S}=$ single code, $\mathrm{T}=$ channel). In these scenarios four, five and seven channels have been used respectively.

Table 1. Enforced scenarios

\begin{tabular}{|c|c|c|c|c|}
\hline & Scenario & 1 & 2 & 3 \\
\hline & & 4 channels & 5 channels & 7 channels \\
\hline 1 & Clock & S & T & T \\
\hline 2 & Init loop & S & S & T \\
\hline 3 & NC & T & T & T \\
\hline 4 & RC & T & T & T \\
\hline 5 & MC & T & T & T \\
\hline 6 & FC & S & S & T \\
\hline 7 & Termination Loop & & & T \\
\hline
\end{tabular}

In the first scenario (fig. 3), only the basic structure procedures are enforced as channels. The restraintler is working as a part of the main application channel and it is active until the counterfeit time termination. When a new counterfeit step starts, the restraintler activates the needed initializations and after the completion the four channels are activated. After completion, each channel sends a signal to the restraintler. When four completion indicators are collected from the restraintler, the final loop task is activated. 


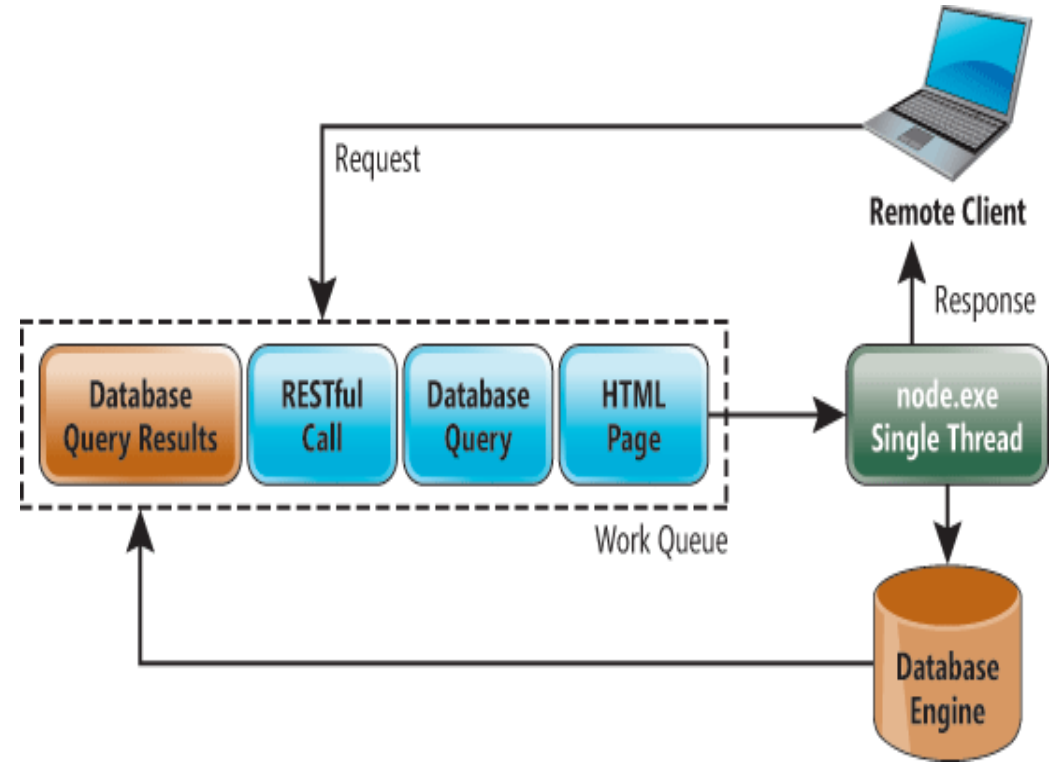

Fig. 3 The 4 channel scenario

The restraintler is converted by channel (fig. 4) in the case of second scenario. While the four channels are executing from JVM, the correlating code within the application is blocked. The restraintler defines the awaking channel between the main counterfeit factors. The last empirical model (fig. 5) consists of seven channels, four channels for the basic structure procedures, one for synchronization and two for supplementary tasks (init loop, final loop). All the enforced channels are always active within the counterfeit time. Special purpose flags inside the body of each channel and in channel with restraint indicators the correlating code is activated. The JVM offers the various approaches for restraintling channels. Using these approach for starting/stopping channels (instead of internal flags) compelling delays and in balance of the counterfeit models can be produced.

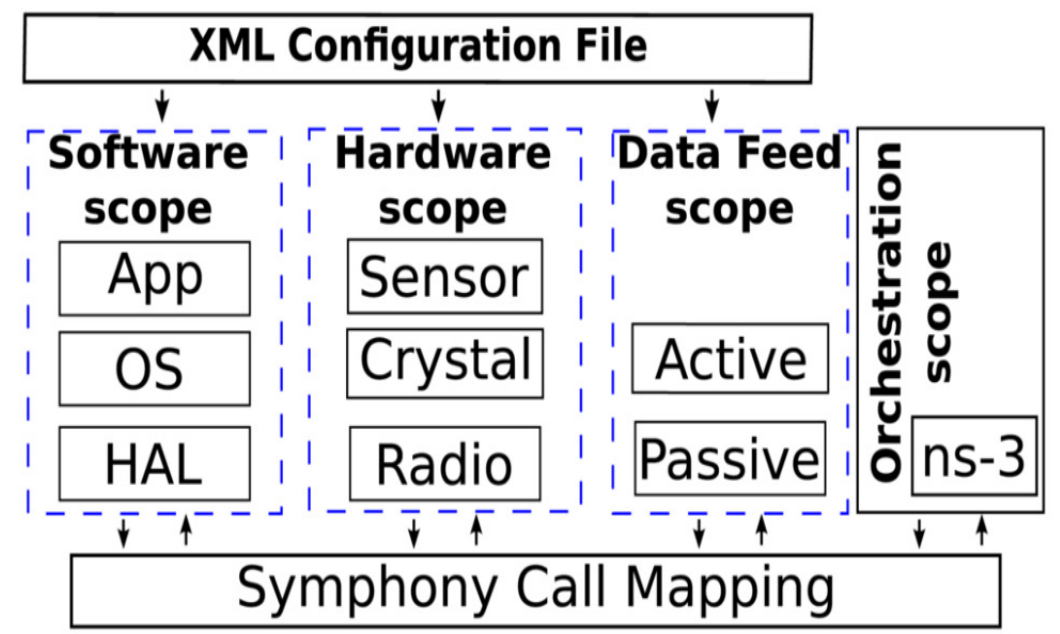

Fig. 4 The 5 channel scenario 


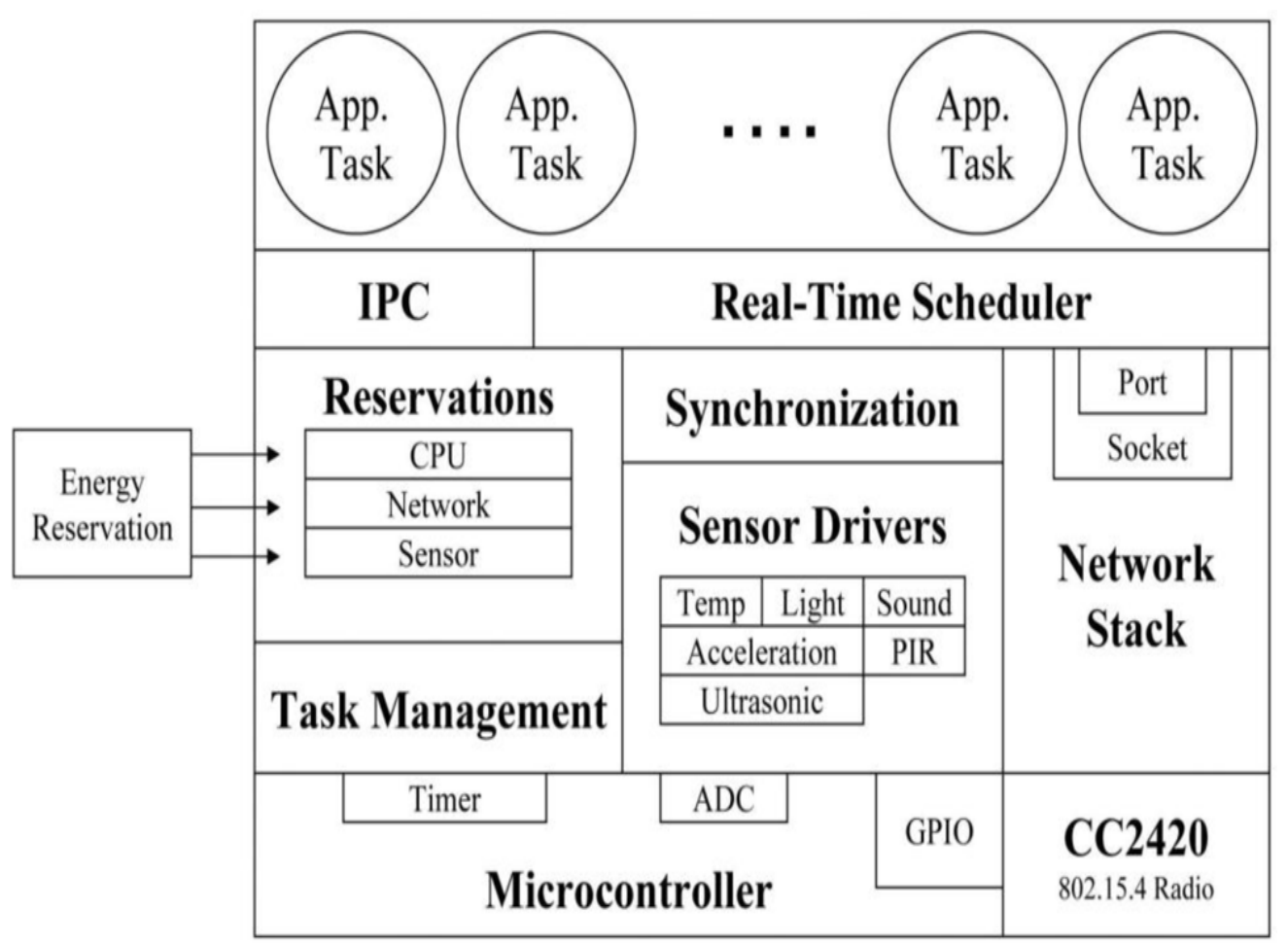

Fig. 5 The 7 channel scenario

\section{DES ORGANIZATION EVALUATIONS}

The major tasks judging accomplishment of a cellular structure are the new call acceptance and handoff (reallocation). This accomplishment can be measured in terms of statistical metrics by using blocking and dropping probability. When a new call acceptance is unsuccessful, then this call is blocked. The blocking probability is calculated from the type:

$$
P_{\text {bloking }}=\frac{\text { number of blocked calls }}{\text { number of calls }}
$$

In a handoff situation, if the structure cannot allocate a new channel for the moving MU, then, this ongoing call is dropped. The correlating probability is calculated as follows:

$$
P_{\text {dropping }}=\frac{\text { number of forced calls }}{\text { number of calls }- \text { number of blocked calls }}
$$

For estimating more accurately the simulating results, Monte Carlo [50] beheadings have been used. 
International Journal of Wireless \& Mobile Networks (IJWMN) Vol. 7, No. 4, August 2015

\section{EMPIRICAL RESULTS}

Figures 6 and 7 show that by increasing channel number, structure accomplishment is augmented

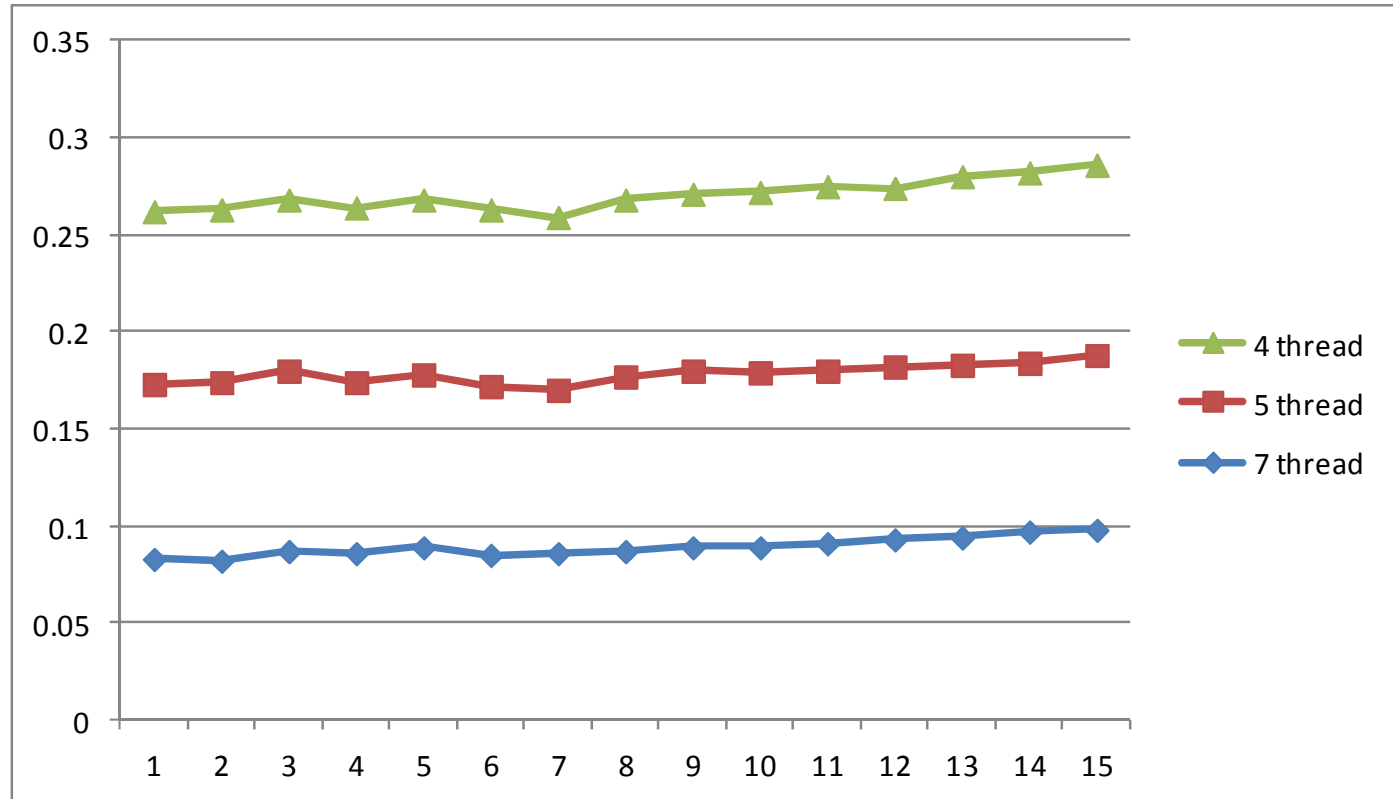

Fig. 6. Blocking anticipation for the involved Architectures

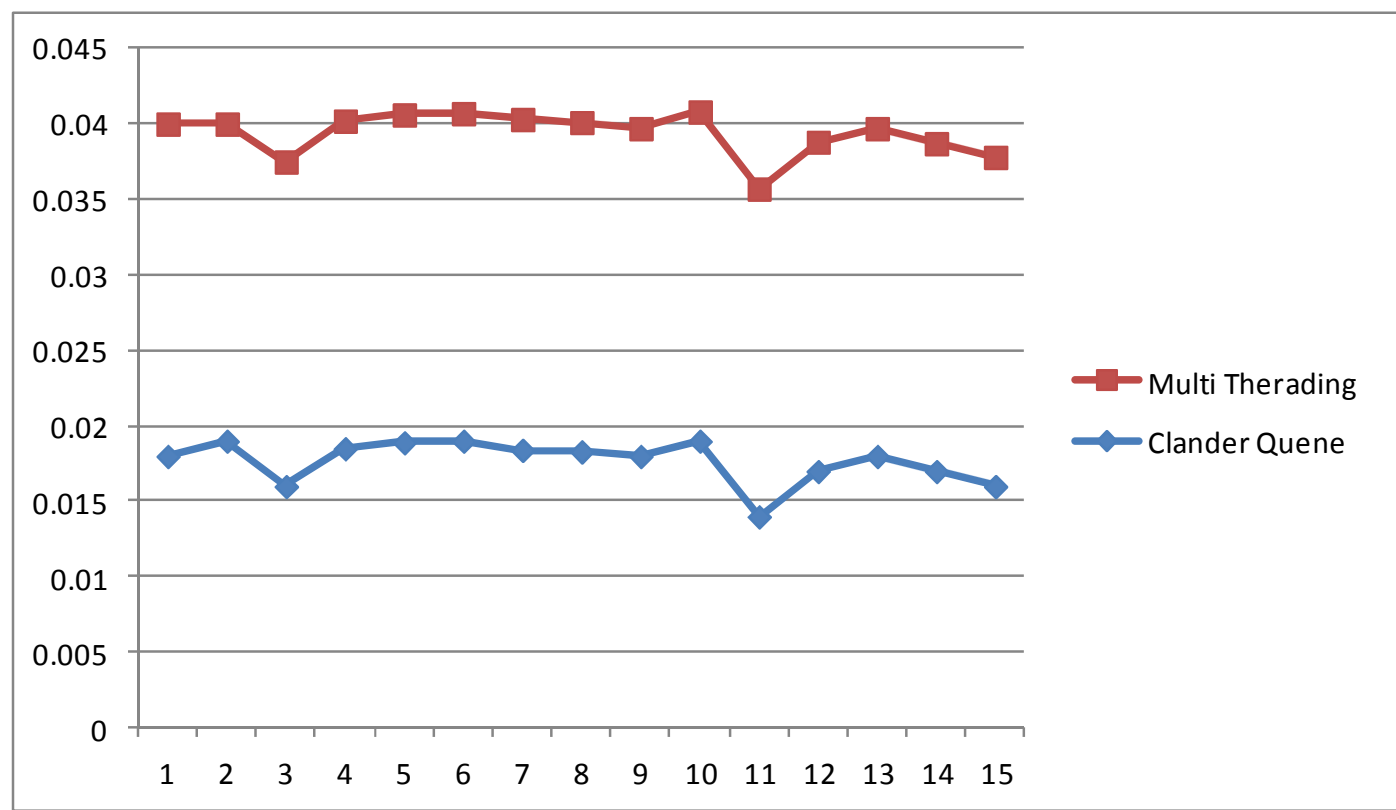

Fig. 7. Dropping anticipation for the CQ and MT accesses 
Tables 2 and 3 confirm the results that are illustrated in previous graphs (figures $8 \& 9$ )

Table 2 Mean values of STD and MEAN for blocking probability

\begin{tabular}{|c|c|c|}
\hline Scenario & \multicolumn{2}{|c|}{ Blocking Mean Value } \\
\hline & STD & MEAN \\
\hline 7 channels & 0.044075 & 0.089216 \\
\hline 5 channels & 0.04387 & 0.080194 \\
\hline 4 channels & 0.044521 & 0.082127 \\
\hline
\end{tabular}

Table 3 Mean values of STD and MEAN for dropping probability

\begin{tabular}{|c|c|c|}
\hline Scenario & \multicolumn{2}{|c|}{ Dropping Mean Values } \\
\hline & STD & MEAN \\
\hline 7 channels & 0.025648 & 0.018489 \\
\hline 5 channels & 0.0357 & 0.022577 \\
\hline 4 channels & 0.033493 & 0.022738 \\
\hline
\end{tabular}

Here I have shown intuitively, the enforced adequate is more pragmatic when the computational time for a counterfeit step is remaining stable (equitable between tasks inside the step). Table 4, shows the ratio std/mean of the counterfeit time duration (ms).

This ratio gives us the advice for the significance of the std of the counterfeit step duration and thus the resulting balance. Data inside table 4, permode results based on Monte Carlo beheadings. The mean values permode the ratio std/mean and the std permodes the standard deviation of the resulted mean values.

Table 4. Counterfeit step duration

\begin{tabular}{|c|c|c|c|}
\hline & 7 channels & 5 channels & 4 channels \\
\hline Mean & 0.055156 & 0.1479 & 0.20057333 \\
\hline Std & 0.03353804 & 0.014321662 & 0.16271174 \\
\hline
\end{tabular}

\section{CONCLUSIONS}

I have conferred in this paper, the con channel of modeled adequate level for the expected counterfeit architectures of wireless communication organizations to counterfeit organization conduct and accomplishment is conferred. Main and basic the concept of the counterfeit step computational duration balance over counterfeit time is illustrated.

It is empirically implied that JVM is in the case of the multi-interweave scenario involving implementation of counterfeit architecture is consisting of four channels and three simple nonchannel tasks, provides equitable time slices only to the four channels. The simple non-channel tasks don't participate this time sharing. The total computational is in time that is needed for each counterfeit step is based on the completion of each individual fundamental such as channels and simple tasks.

In the case of less than seven channels implementation the total time is asymmetrically allocated to the factors and so no equitable conditions could be guaranteed as the main role of the mission. When the mentioned factors are all channels, the time slicing that is provided by JVM is fairly allocated among the active channels and so equitable conditions are created. The empirical results are shown that there is high positive correlation between organization accomplishment and counterfeit time balance implying pragmatic reflection of the Physical Structure Time. 
There is no doubt that the JVM environment complements an effective device for developing a multi interweaves environment and its pure uses. On the other hand, analytical drawbacks of the JVM access (e.g. deadlocks, compelling delays due to channel priorities, synchronization problems, etc) should be effectively faced by the developer. The major imminent research work towards developing an adequate counterfeit model for wireless communication organizations is to focus on balancing drawbacks and benefits of such a JVM based app.

\section{REFERENCES}

[1] Reuben Pasquini, Algorithms for improving the achievement of optimistic parallel counterfeit, $\mathrm{PhD}$ dissertation, Purdue University, 1999

[2] Dirk Brade, A generalized process for the verification and validation of models and counterfeit results, PhD. Dissertation, university of Bundeswehr Munchen, 2003

[3] Rimon Barr, An adequate, unifying access to counterfeit using virtual machines, Cornell University, 2004

[4] R. S. M. GOH and I. L-J THNG, MLIST: An adequate pending event set structure for distinct event counterfeit, International Journal of Counterfeit Vol. 4 No. 5-6, 2003

[5] Gianni A. Di Caro, Analysis of counterfeit environments for mobile ad hoc networks, Technical Report No. IDSIA-24-03, Dalle Molle Institute for Artificial Intelligence, 2003

[6] Thomas J. Schreiber, Daniel T. Brunner, Inside Distinct-Event Counterfeit Software: How It Works and Why It Matters, Proceedings of the 1997 Winter Counterfeit Conference, 1997

[7] Jayadev Misra, Distributed Distinct-event Counterfeit, ACM, Computing Surveys, Vol. 18, No. 1, March 1986

[8] Benno Jaap Overeinder, Distributed Event-driven Counterfeit, $\mathrm{PhD}$ dissertation, University of Amsterdam, 2000

[9] Bruno R. Preis, Wayne M. Loucks, V. Carl Hamacher, A Unified Modeling Methodology for Achievement Evaluation of Distributed Distinct Event Counterfeit Apparatus Structure S, the 1988 Winter Counterfeit Conference, 1988

[10] Kalyan S. Perumalla, Parallel and Distributed Counterfeit: Traditional Method and Recent Advances, Proceedings of the 2006 Winter Counterfeit Conference, 2006

[11] Mathieu Lacage, Thomas R. Henderson, yet another Network Simulator, ACM 2006

[12] Rick Siow Mong Goh, Ian Li- Jin Thng, DSplay: An Adequate Dynamic Priority Queue Structure for Distinct Event Counterfeit,

[13] Kehsiung Chung, Janche sang and Vernon rego, A Achievement Comparison of Event Calendar Algorithms: an Empirical Access, Software-Practice and Experience, Vol. 23(10), 1107- 1138 (October 1993)

[14] Lucite Muliadi, Distinct Event Modeling In .tolemy II, University of California, Berkeley, 1999

[15] Valerie Naoumov, Thomas Gross Counterfeit of Large Ad Hoc Networks, MSWiM'03, September, San Diego, California, USA, 2003

[16] Kevin Fall, Kannan Varadhan, The ns Manual, UC Berkeley, LBL, USC/ISI, and Xerox PARC, January 14, 2007

[17] Stuart Kurkowski, Tracy Camp, and Michael Colagrosso, MANET Simulation Studies: The Current State and New Simulation Tools, 2005

[18] Xiang Zeng, Rajive Bagrodia, Mario Gerla, "GloMoSim: A Library for Parallel Simulation of Largescale Wireless Networks", Proceedings of the 12th Workshop on Parallel and Distributed Simulations, 1998

[19] Lokesh Bajaj, Mineo Takai, Rajat Ahuja, Rajive Bagrodia, "Simulation of Large-Scale Heterogeneous Communication Systems", Proceedings of IEEE Military Communications Conference (MILCOM'99), 1999

[20] A. Boukerche, S. K. Das, A. Fabbri, "Swim Net: A Scalable Parallel Simulation Test bed for Wireless and Mobile Networks", Wireless Networks 7, 467-486, 2001

[21] Winston Liu, Changchun Chiang, Hsiao_Kuang Wu, Vikas Jha, Mario Gerla, Rajive Bagrodia, "Parallel Simulation Environment for Mobile Wireless NetworkS", Proceedings of the 1996 Winter Simulation Conference, WSC'96, pp. 605- 612,1996

[22] Richard M. Fujimoto. Parallel and Distributed Simulation Systems. Parallel and Distributed Computing. Wiley-Interscience, 2000. 
[23] Kevin Fall, Kannan Varadhan, (2007), The ns Manual, UC Berkeley, LBL, USC/ISI, and Xerox PARC, January 14.

[24] Lukito Muliadi, (1999), Discrete Event Modeling In Ptolemy Ii, Department of Electrical Engineering and Computer Science University of California, Berkeley.

[25] http://jist.ece.cornell.edu/javadoc/jist/runtime/Scheduler.Calendar.html

[26] Rick Siow Mong Goh, Ian Li- Jin Thng, (2004), DSplay: An Efficient Dynamic Priority Queue Structure for Discrete Event Simulation, SimTech Simulation Technology and Training Conference, Australia

[27] R. S. M. GOH and I. L-J THNG, (2003), MLIST: An Efficient Pending Event Set Structure For Discrete Event Simulation, International Journal of Simulation Vol. 4 No. 5-6.

\section{Author}

Rajeev Ranjan received his B.E degree in Electronics and Telecommunication from Swami Ramananad Teerth Marathwada University, Nanded, Maharashtra, India in 1999, and M.E degree in Electronics and Communication from BIT Mesra, Ranchi, Jharkhand, India in 2002. He is currently pursuing PhD from the Department of Electronics \& Communication, in university of Banasthali. His research interest's area is ad-hoc networks, algorithm design in the wireless network and analog communication.

Dr. Praveen Dhyani is Professor of Computer Science and Executive Director at Banasthali University Jaipur Campus. Previously he was the Rector of the Birla Institute of Technology, MESRA; prior to which he held key academic cum administrative positions in BITS Pilani, and BIT, MESRA. He has supervised doctoral research and has authored research papers, technical reports, and international conference proceedings in diverse fields of Computer Science and Information Technology. His R\&D accomplishments

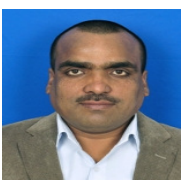
include development and national and international exhibit of robot, development of electronics devices to aid foot drop patients, and development of voice operated wheel chair. He is presently member of the academic and research regulatory bodies of the Banasthali University, and the departmental research committee of IIS University, India."

Dr. Rajeev Kumar is Assistant Professor in the Department of Electronics \& Communication Engineering at Waljat College of Applied Sciences, Muscat, Sultanate of Oman since November 2010. Before joining the Waljat College he was Assistant Professor in the Department of Applied Physics, Birla Institute of Technology, Mesra, Ranchi, India. Rajeev Kumar obtained his Master's degree in Physics from Indian Institute of Technology, Madras, India and Ph. D. from University of Calcutta, Kolkata, India. His research interests are in the areas of Experimental Plasma Physics, Spectral Analysis and Numerical Methods.

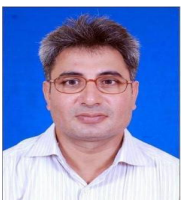

